\title{
Sensitivity of Legged Balance Control to Uncertainties and Sampling Period
}

\author{
Nahuel A. Villa ${ }^{1}$, Johannes Englsberger ${ }^{2}$ and Pierre-Brice Wieber ${ }^{1}$
}

\begin{abstract}
We propose to quantify the effect of sensor and actuator uncertainties on the control of the center of mass and center of pressure in legged robots, since this is central for maintaining their balance with a limited support polygon. Our approach is based on robust control theory, considering uncertainties that can take any value between specified bounds. This provides a principled approach to deciding optimal feedback gains. Surprisingly, our main observation is that the sampling period can be as long as $200 \mathrm{~ms}$ with literally no impact on maximum tracking error and, as a result, on the guarantee that balance can be maintained safely. Our findings are validated in simulations and experiments with the torquecontrolled humanoid robot Toro developed at DLR. The proposed mathematical derivations and results apply nevertheless equally to biped and quadruped robots.
\end{abstract}

Index Terms-Legged robots, Balance control, Robust control, Sampling period, Bounded input bounded output.

\section{INTRODUCTION}

B IPED and quadruped robots are beginning now to master the skill of walking dynamically in most standard situations [2], [4], [9]. This suggests that more widespread commercial use of such robots will soon be possible. This requires, however, that guarantees are provided about their safety and operational performance. In research prototypes, the risk of failure is usually contained by using very fast and precise (and therefore very expensive) sensors, actuators and computers, resulting in robots that are clearly too expensive for commercial purposes.

The dynamics of the Center of Mass (CoM) of these robots over the support feet is unstable, and therefore very sensitive to all sources of uncertainties. But how fast and precise, and therefore how expensive should the sensors, actuators and computers be has never been investigated in the existing scientific literature. A precise quantification of the effect of uncertainties and sampling period on legged balance control seems to be missing, and it is the goal of this paper to initiate this discussion.

The balance of legged robots mostly involves motion of their $\mathrm{CoM}$ with respect to their feet on the ground. We therefore

Manuscript received: February 24, 2019; Revised June 22, 2019 Accepted June 29, 2019. This paper was recommended for publication by Editor Nikos Tsagarakis upon evaluation of the Associate Editor and Reviewers' comments.

This work was funded by the EU H2020 Comanoid Research and Innovation Action (RIA).

${ }^{1}$ Univ. Grenoble Alpes, Inria, 38000 Grenoble, France nahuel.villadinria.fr;

pierre-brice.wiebereinria.fr.

${ }^{2}$ German Aerospace Center (DLR), Institute of Robotics and Mechatronics, 82234 Wessling, Germany. johannes. englsbergerdalr. de

Digital Object Identifier (DOI): see top of this page.

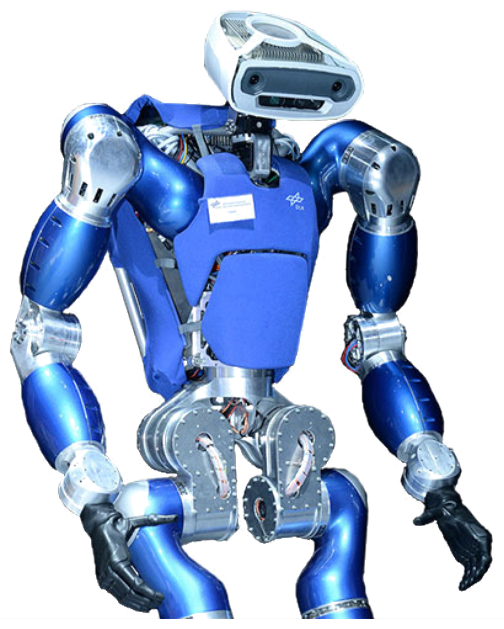

Fig. 1. Toro is a torque-controlled humanoid robot developed at DLR [3].

focus our analysis on the motion of the CoM, considering that other aspects of the motion of the robot, such as precise whole-body joint motion and contact force control, are handled separately, as usual in this field of robotics [15].

We introduced in [14] a tube-based Model Predictive Control (MPC) of walking in order to guarantee that all kinematic and dynamic constraints are always satisfied, even in the presence of uncertainties. We considered that uncertainties can take any value between some bounds, generating some tracking error which can be bounded accordingly. Here, we propose to analyse how these bounds are related: how much tracking error can we expect for a given amount of uncertainty? This naturally depends not only on the kind of uncertainty (e.g. on sensors or actuators), but also on the control law and its sampling period.

Our findings are validated in experiments and simulations with the torque-controlled humanoid robot Toro developed at DLR (Fig. 1). The proposed mathematical derivations and results apply nevertheless indistinctly to biped and quadruped robots.

Section II introduces basic aspects of the CoM dynamics. A standard feedback control law is proposed and conditions for its stability are determined in Section III. The dynamics of the tracking error is analysed in Section IV and related to the bounded uncertainties in Section V. Feedback gains are then optimized to minimize the span of the tracking error in Section VI. Our theoretical analysis is validated experimentally and in simulations on the torque-controlled humanoid robot Toro in Section VII. Finally, we summarize our conclusions in Section VIII. 


\section{WALKING MODEL}

Consider a legged robot walking on flat, horizontal ground. The Center of Pressure (CoP) $p$ of the contact forces with the ground can be related to the motion of the Center of Mass $(\mathrm{CoM}) c$ of the robot and its angular momentum $L$ as follows [15]:

$$
p^{x, y}=c^{x, y}-\frac{m c^{z} \ddot{c}^{x, y}-S \dot{L}^{x, y}}{m\left(\ddot{c}^{z}+g^{z}\right)},
$$

where ${ }^{x}$ and ${ }^{y}$ indicate horizontal coordinates, $g^{z}$ is the vertical acceleration due to gravity, $m$ the mass of the robot and $S=\left[\begin{array}{cc}0 & -1 \\ 1 & 0\end{array}\right]$ a $\frac{\pi}{2}$ rotation matrix. Due to the unilaterality of contact forces, this CoP is bound to the support polygon $\mathcal{P}(t)$, which varies with time depending on which feet are in contact with the ground and where:

$$
p \in \mathcal{P}(t) .
$$

This can be reformulated as a dynamics

$$
\ddot{c}^{x, y}=\omega^{2}\left(c^{x, y}-p^{x, y}+n^{x, y}\right),
$$

with some constant value $\omega^{2} \approx \frac{g^{z}}{c^{z}}$, gathering all nonlinearities in a vector

$$
n=\frac{\ddot{c}}{\omega^{2}}-\frac{m c^{z} \ddot{c}-S \dot{L}}{m\left(\ddot{c}^{z}+g^{z}\right)},
$$

which can be bounded efficiently [1], [12]:

$$
n \in \mathcal{N} \text {. }
$$

Since the ${ }^{x}$ and ${ }^{y}$ coordinates appear to be decoupled, we will consider only the ${ }^{x}$ coordinate in the following.

Assuming that $p$ and $n$ are constant over time intervals of length $\tau$, we can obtain a discrete-time linear system following a standard procedure [11]:

$$
x^{+}=A x+B(p-n),
$$

with matrices

$$
\begin{aligned}
& A=\left[\begin{array}{cc}
\cosh (\omega \tau) & \omega^{-1} \sinh (\omega \tau) \\
\omega \sinh (\omega \tau) & \cosh (\omega \tau)
\end{array}\right], \\
& B=\left[\begin{array}{c}
1-\cosh (\omega \tau) \\
-\omega \sinh (\omega \tau)
\end{array}\right],
\end{aligned}
$$

and $x^{+}$the successor of the state

$$
x=\left[\begin{array}{c}
c^{x} \\
\dot{c}^{x}
\end{array}\right] \in \mathcal{X}(t),
$$

where $\mathcal{X}(t)$ represents time-varying kinematic constraints on the CoM motion.

\section{Stable Feedback Gains}

We control the CoP $p$ using a linear feedback with compensation of $n$ :

$$
p=p_{\text {ref }}+K\left(x-x_{r e f}\right)+n
$$

with feedback gains of the form

$$
K=k\left[\begin{array}{ll}
1 & \lambda
\end{array}\right]
$$

in order to track a reference trajectory $x_{r e f}, p_{r e f}$ (obtained with any standard motion generation scheme [15]). If the reference trajectory follows the dynamics (6) without $n$ :

$$
x_{r e f}^{+}=A x_{r e f}+B p_{r e f},
$$

this leads to a closed-loop dynamics

$$
\tilde{x}^{+}=(A+B K) \tilde{x}
$$

of the tracking error

$$
\tilde{x}=x-x_{\text {ref }} .
$$

Consider the two poles, $q_{1}$ and $q_{2}$, related to this closed-loop dynamics as follows:

$$
\begin{aligned}
q_{1} q_{2} & =\operatorname{det}(A+B K) \\
& =1-k+k \cosh (\omega \tau)-k \lambda \omega \sinh (\omega \tau), \\
q_{1}+q_{2} & =\operatorname{tr}(A+B K) \\
& =k+(2-k) \cosh (\omega \tau)-k \lambda \omega \sinh (\omega \tau) .
\end{aligned}
$$

Following Jury's simplified stability criterion [8], this closedloop dynamics is stable if and only if:

$$
\begin{array}{r}
q_{1} q_{2}<1, \\
\left(q_{1}-1\right)\left(q_{2}-1\right)=q_{1} q_{2}-\left(q_{1}+q_{2}\right)+1>0, \\
\left(q_{1}+1\right)\left(q_{2}+1\right)=q_{1} q_{2}+\left(q_{1}+q_{2}\right)+1>0,
\end{array}
$$

which corresponds to the following constraints represented in Fig. 2:

$$
\begin{aligned}
\lambda & >\frac{\cosh (\omega \tau)-1}{\omega \sinh (\omega \tau)}, \\
k & >1 \\
k \lambda & <\frac{\cosh (\omega \tau)+1}{\omega \sinh (\omega \tau)} .
\end{aligned}
$$

\section{From UnCERTAinties to TRACKING ERROR}

Consider that the CoP is affected by a bounded additive uncertainty

$$
v \in W
$$

coming from actuators, sensors and model errors (a precise expression will be introduced in Section VI), such that the $\mathrm{CoP}$ in the linear feedback (10) actually is:

$$
p=p_{\text {ref }}+K\left(x-x_{\text {ref }}\right)+n+v .
$$

The closed-loop dynamics (13) becomes

$$
\tilde{x}^{+}=(A+B K) \tilde{x}+B v .
$$

If the closed-loop matrix $A+B K$ is stable, then when time tends to infinity, the tracking error $\tilde{x}$ converges to a set $Z$ :

$$
\tilde{x} \rightarrow Z=\bigoplus_{i=0}^{\infty}(A+B K)^{i} B W,
$$

where the symbol $\oplus$ represents a Minkowski sum ${ }^{1}$. Following (25) and (26), once the tracking error is in $Z$, it stays in $Z$ for every future time [10]:

$$
\forall v \in W, \tilde{x} \in Z \Longrightarrow \tilde{x}^{+} \in Z .
$$

\footnotetext{
${ }^{1}$ Given sets $A$ and $B, A \oplus B=\{a+b \mid a \in A, b \in B\}$.
} 


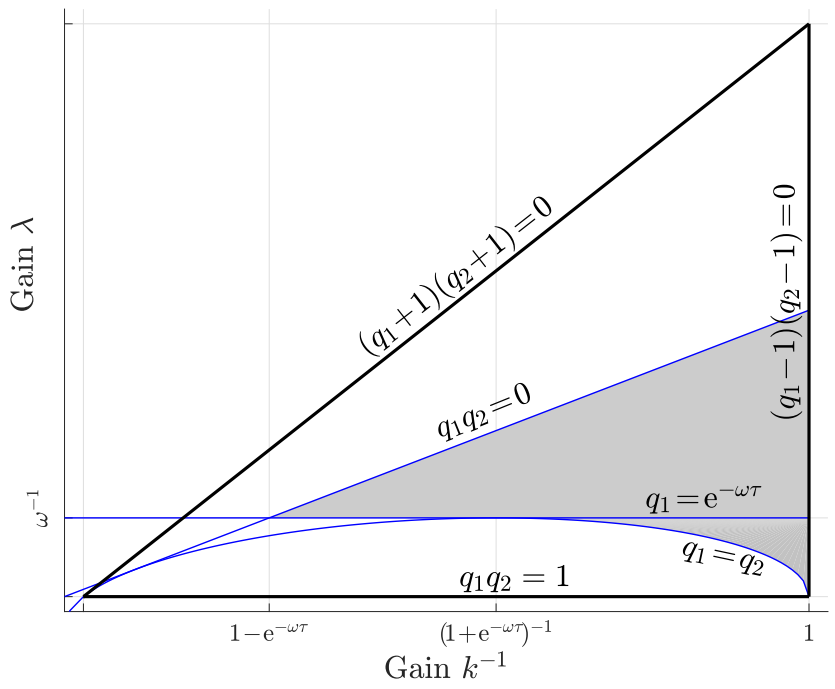

Fig. 2. Following Jury's simplified stability criterion, the region of feedback gains $k^{-1}$ and $\lambda$ that lead to a stable closed-loop dynamics is a triangle, where $\lambda$ varies between $\frac{\cosh (\omega \tau)-1}{\omega \sinh (\omega \tau)}$ and $\frac{\cosh (\omega \tau)+1}{\omega \sinh (\omega \tau)}$ while $k^{-1}$ varies between $\frac{\cosh (\omega \tau)-1}{\cosh (\omega \tau)+1}$ and 1 . The thin blue lines correspond to having both poles equal, at least one pole equal to $\mathrm{e}^{-\omega \tau}$ (when $\lambda=\omega^{-1}$ ), or at least one pole equal to zero. The gray area corresponds to having both poles positive real, and at least one greater or equal to $\mathrm{e}^{-\omega \tau}$.

We use this robust positive invariance property to ensure a bounded tracking error

$$
\tilde{x} \in Z,
$$

provided that the robot motion starts within these bounds. As an example, the tube-based MPC scheme proposed in [14] for biped walking generates the reference motion $x_{r e f}$ online under this condition.

This precise bound on the tracking error allows guaranteeing that the kinematic constraint (9) will always be satisfied, even with the uncertainty (23), provided that

$$
x_{r e f} \in \mathcal{X}(t) \ominus Z,
$$

where the symbol $\ominus$ represents a Pontryagin difference ${ }^{2}$. In this case, the corresponding $\mathrm{CoP}$ tracking error

$$
\tilde{p}=p-p_{\text {ref }}-n=K \tilde{x}+v
$$

is bounded accordingly:

$$
\tilde{p} \in K Z \oplus W,
$$

so we can guarantee that the support polygon constraint (2) will be satisfied as well, provided that

$$
p_{\text {ref }} \in \mathcal{P}(t) \ominus \mathcal{N} \ominus K Z \ominus W .
$$

Feasibility of the reference motion generation and tracking imposes that the sets in (29) and (32) are non-empty. The support polygon $\mathcal{P}(t)$ constraining the $\mathrm{CoP}$ is normally smaller than the kinematic constraints $\mathcal{X}(t)$ on the CoM motion. And the bound $K Z \oplus W$ on the CoP tracking error is larger than the

\footnotetext{
${ }^{2}$ Given sets $A$ and $B, A \ominus B=\{x \mid x+B \subseteq A\}$.
}

bound $Z$ on the CoM tracking error when using stable gains $K$, satisfying condition (21). Thus, as usual in the balance of legged robots, the constraint (32) on the $\mathrm{CoP}$ is the limiting factor, and we look to reduce specifically the bound $K Z \oplus W$ on the $\mathrm{CoP}$ tracking error.

\section{CoP Tracking ERror DUE to UnCERTAinties}

Using definition (26) of the set $Z$, the bound (31) on the $\mathrm{CoP}$ tracking error becomes:

$$
\tilde{p} \in \bigoplus_{i=0}^{\infty} K(A+B K)^{i} B W \oplus W .
$$

Considering a real interval

$$
W=\left[v_{\min }, v_{\max }\right],
$$

the maximum and minimum values for $\tilde{p}$ are reached with opposite sequences of maximum and minimum values $v_{\max }$ and $v_{\text {min }}$, depending on the sign of each real coefficient $K(A+B K)^{i} B$. This results in

$\tilde{p}_{\text {max }}-\tilde{p}_{\min }=\left(\sum_{i=0}^{\infty}\left|K(A+B K)^{i} B\right|+1\right)\left(v_{\max }-v_{\min }\right)$.

We can introduce then the spans

$$
\tilde{p}_{\text {span }}=\tilde{p}_{\max }-\tilde{p}_{\text {min }}
$$

and

$$
v_{\text {span }}=v_{\max }-v_{\min },
$$

and the ratio

$$
r=\frac{\tilde{p}_{\text {span }}}{v_{\text {span }}}=\sum_{i=0}^{\infty}\left|K(A+B K)^{i} B\right|+1
$$

between the amount of uncertainty and the resulting amount of CoP tracking error.

The gray area in Fig. 2 corresponds to having both poles $q_{1}$ and $q_{2}$ positive real, and at least one greater or equal to $\mathrm{e}^{-\omega \tau}$. The extent of this area depends on the product $\omega \tau$, but inside this area, the above ratio is

$$
r=\frac{1}{k-1}+2,
$$

as shown in the Appendix, which is surprisingly independent from $\lambda, \omega$ and $\tau$. It can be observed numerically that this is actually the minimum possible ratio. Within this area, the choice $\lambda=\omega^{-1}$ is particularly interesting since it maximizes controllability [13]. We consider therefore feedback gains of the form:

$$
K=k\left[1 \quad \omega^{-1}\right] .
$$

In that case, we obtain poles

$$
\begin{aligned}
& q_{1}=\mathrm{e}^{-\omega \tau}, \\
& q_{2}=1-(k-1)\left(\mathrm{e}^{\omega \tau}-1\right)
\end{aligned}
$$

from (15) and (16). Borrowing from the Appendix the reformulation (59) of the infinite sum (38) with coefficients (65) and (66), we obtain that the above ratio becomes

$$
r=\frac{k\left(\mathrm{e}^{\omega \tau}-1\right)}{1-\left|q_{2}\right|}+1 \text {. }
$$


Depending on the sign of $q_{2}$ (positive being inside of the gray area, negative being outside), we have:

$$
r= \begin{cases}\frac{1}{k-1}+2 & \text { if } \mathrm{e}^{\omega \tau}-1 \leq \frac{1}{k-1}, \\ \frac{2+\left(\mathrm{e}^{\omega \tau}-1\right)}{2-(k-1)\left(\mathrm{e}^{\omega \tau}-1\right)} & \text { if } \frac{1}{k-1} \leq \mathrm{e}^{\omega \tau}-1<\frac{2}{k-1} .\end{cases}
$$

When $\mathrm{e}^{\omega \tau}-1 \geq \frac{2}{k-1}$, the closed loop is unstable and the ratio $r$ is undefined.

\section{Optimal Gains And SAMPling Periods}

With feedback gains of the form (40), the linear feedback with uncertainties (24) can be reformulated as

$$
p=p_{r e f}+k\left(\xi-\xi_{r e f}\right)+n+v,
$$

where

$$
\xi=c+\omega^{-1} \dot{c}
$$

is the Capture Point (CP) [15]. Considering an error $\hat{\xi}$ in the estimation of the CP $\xi$ and an error $\hat{n}$ in the model of the robot (including inaccuracies in the actuation and ground contact), this linear feedback actually becomes

$$
p=p_{\text {ref }}+k\left(\xi+\hat{\xi}-\xi_{\text {ref }}\right)+n+\hat{n},
$$

corresponding to an uncertainty $v$ of the form:

$$
v=k \hat{\xi}+\hat{n} .
$$

Using the ratio (38), the resulting span of $\mathrm{CoP}$ tracking error is:

$$
\tilde{p}_{\text {span }}=r k \hat{\xi}_{\text {span }}+r \hat{n}_{\text {span }} .
$$

Based on (44), its minimum value

$$
\tilde{p}_{\text {span }}^{*}=\left(\sqrt{\hat{\xi}_{\text {span }}}+\sqrt{2\left(\hat{\xi}_{\text {span }}+\hat{n}_{\text {span }}\right)}\right)^{2}
$$

is obtained using a feedback gain

$$
k^{*}=1+\sqrt{\frac{\hat{\xi}_{\text {span }}+\hat{n}_{\text {span }}}{2 \hat{\xi}_{\text {span }}}} .
$$

Typical values for these sources of uncertainties are $\hat{n}_{\text {span }}=$ $\hat{\xi}_{\text {span }}=1 \mathrm{~cm}$ [5], resulting in a minimal span of CoP tracking error $\tilde{p}_{\text {span }}^{*}=9 \mathrm{~cm}$, corresponding to Toro's feet width. In this case, the optimal gain is $k^{*}=2$.

But the key observation in (44) is that once a gain $k$ has been decided, the ratios $r$ and $r k$ don't depend on the sampling period $\tau$, as long as it is shorter than

$$
\tau_{0}=\omega^{-1} \ln \left(\frac{1}{k-1}+1\right) .
$$

The maximum CoP tracking error $\tilde{p}_{\text {span }}$ is not improved by reducing the sampling period below this value, but it degrades sharply when $\tau>\tau_{0}$, as shown in Fig. 3 . When $k=2$, $\tau_{0}=\omega^{-1} \ln 2=216 \mathrm{~ms}\left(\omega \approx 3.2 \mathrm{~s}^{-1}\right.$ for Toro $)$.

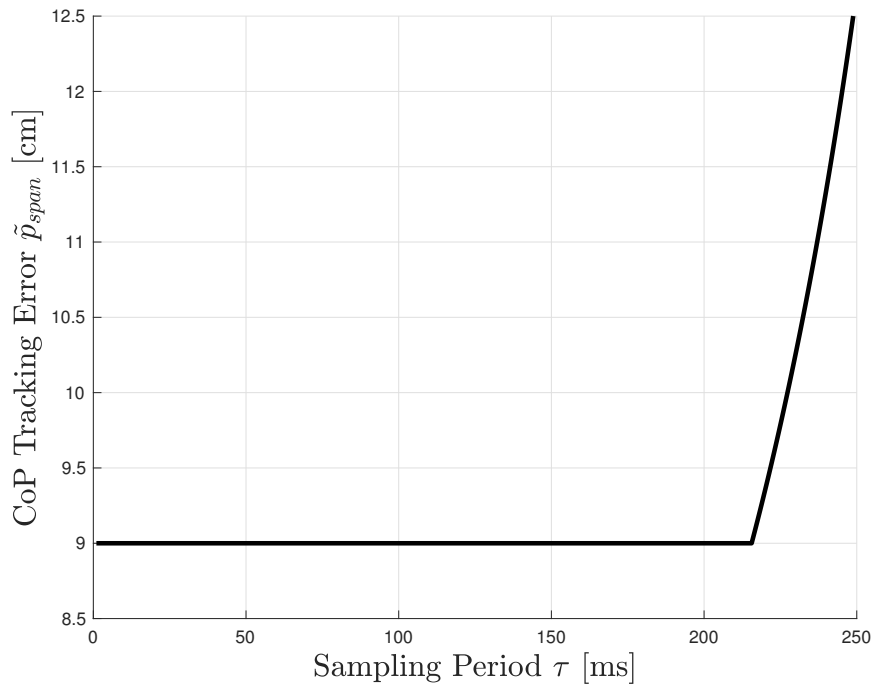

Fig. 3. Span of the CoP tracking error $\tilde{p}_{\text {span }}$ produced by model and estimation errors with span $\hat{n}_{\text {span }}=\hat{\xi}_{\text {span }}=1 \mathrm{~cm}$, using the optimal feedback gains $k=2$ and $\lambda=\omega^{-1}\left(\omega \approx 3.2 \mathrm{~s}^{-1}\right.$ for Toro) for different sampling periods $\tau$. The tracking error degrades sharply when $\tau>\omega^{-1} \ln 2=216 \mathrm{~ms}$, but it doesn't improve for sampling periods below this value.

\section{EXPERIMENTAL RESULTS}

The CP linear feedback (45) is implemented in the humanoid robot Toro, with a feedback gain $k=2$ as discussed above, together with a standard Quadratic Program (QP) based inverse dynamics scheme for Whole-Body Control (WBC) of joint positions and contact forces [3]. The sampling period of the QP-based WBC is kept constant at $3 \mathrm{~ms}$ while varying the sampling period $\tau$ of the $\mathrm{CP}$ feedback (45). The reference trajectory for a simple sequence of steps is actually not adapted to the sampling period, making it more difficult to track precisely at each contact transition with longer sampling periods (see video).

We can observe in Fig. 4 that in experiments with Toro, the lateral $\mathrm{CP}$ and $\mathrm{CoP}$ tracking performances are similar and satisfactory when $\tau=51 \mathrm{~ms}$ or $120 \mathrm{~ms}$, as expected from our theoretical analysis. For longer sampling periods, the WBC generates larger arm motions in order to compensate angular momentum variations, which ends up triggering an emergency stop due to the increased risk of collision (see video). The resulting failure originates in the QP-based WBC and not the CP linear feedback (45), so this doesn't contradict the proposed theoretical analysis. In simulations, this safety system is not triggered and we can observe in Fig. 5 that the tracking performance is maintained at a satisfactory level for sampling periods up to $\tau=216 \mathrm{~ms}$ while degrading sharply afterwards, validating strikingly well the theoretical analysis proposed above.

\section{DISCUSSION AND CONCLUSION}

We quantify the effect of sensor and actuator uncertainties on the CoM and CoP tracking error in legged robots, since this is central for maintaining their balance with a limited support 

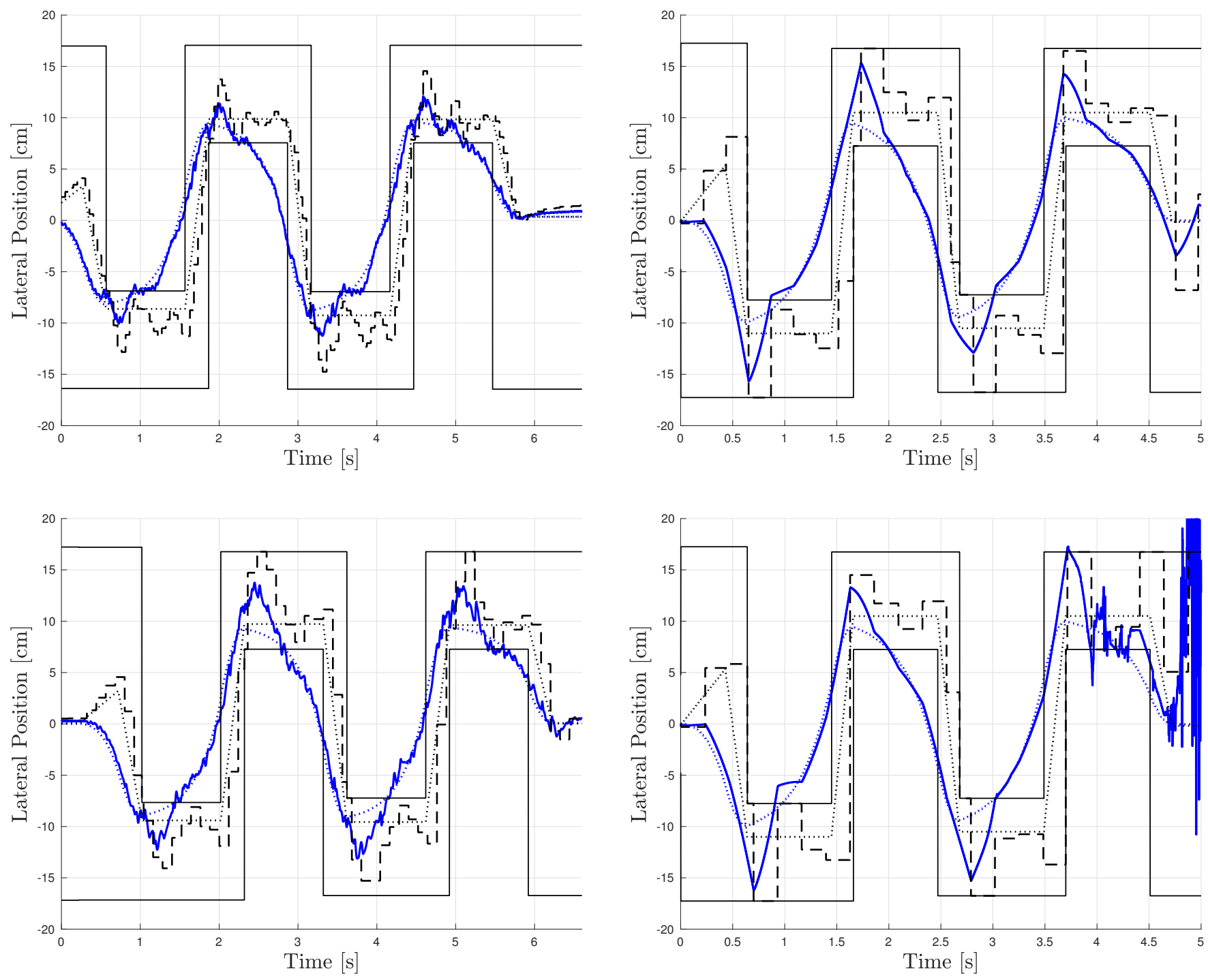

Fig. 4. Lateral component of walking experiments with the humanoid robot Toro using a feedback gain $k=2$ and sampling period $\tau=51 \mathrm{~ms}$ (top) or $\tau=120 \mathrm{~ms}$ (bottom). The $\mathrm{CP} \xi$ is represented in blue, while the $\mathrm{CoP}$ is in dashed black. The reference values $\xi_{\text {ref }}$ and $p_{\text {ref }}$ are indicated with dotted lines.

polygon. Our approach is based on robust control theory, considering uncertainties that can take any value between some bounds. The relationships we obtain can be used during the design stage of a legged robot, when looking for the best compromise between sensor, actuator, and CPU performance and cost. This principled approach also provides the corresponding optimal feedback gains.

Our main observation is that the sampling period for a human-sized humanoid robot such as Toro can be as long as $200 \mathrm{~ms}$ with literally no impact on maximum tracking error and, as a result, on the guarantee that balance can be maintained safely. Concerning quadruped robots, stable locomotion has been realized recently with similarly low, $15 \mathrm{~Hz}$ control rates [6]. Faster sampling periods might be useful for other aspects of the motion of the robot, such as arm or swing leg motion, but not for CoM motion.

Fig. 5. Lateral component of walking simulations with the humanoid robot Toro using a feedback gain $k=2$ and sampling periods $\tau=216 \mathrm{~ms}$ (top) or $\tau=232 \mathrm{~ms}$ (bottom). The $\mathrm{CP} \xi$ is represented in blue, while the CoP is in dashed black. The reference values $\xi_{\text {ref }}$ and $p_{\text {ref }}$ are indicated with dotted lines.

This provides some freedom in the choice of the sampling period, which helped us achieve a substantial reduction of the oscillations mentioned in [3] by avoiding structure resonance modes. This could also help reduce energy consumption, using lower gains, estimating the state and computing the control law less often (CPU power consumption has been observed to represent a significant fraction of the whole power consumption of the robot Toro [7]).

The proposed analysis doesn't consider maintaining balance by actively using angular momentum (whirling limbs in the air) or modifying the support polygon by making a step. Investigating how uncertainties relate to the decision to make steps, when, how and where, is our next goal. 


\section{APPENDIX}

If each real coefficient $K(A+B K)^{i} B$ in the infinite sum (38) is negative, we actually have

$$
r=-K h+1,
$$

where

$$
h=\sum_{i=0}^{\infty}(A+B K)^{i} B .
$$

By construction, this vector $h$ is the solution of

$$
h=(A+B K) h+B,
$$

which can be easily obtained:

$$
h=\left[\begin{array}{c}
\frac{1}{1-k} \\
0
\end{array}\right],
$$

resulting in a ratio

$$
r=\frac{1}{k-1}+2
$$

independent from $\lambda, \omega$ and $\tau$.

In order to show that this is the case in the gray area of Fig. 2, factorize the closed-loop matrix as follows:

$$
A+B K=M\left[\begin{array}{cc}
q_{1} & 0 \\
0 & q_{2}
\end{array}\right] M^{-1},
$$

with an invertible matrix $M$, so that:

$$
\begin{aligned}
r & =\sum_{i=0}^{\infty}\left|K M\left[\begin{array}{cc}
q_{1}^{i} & 0 \\
0 & q_{2}^{i}
\end{array}\right] M^{-1} B\right|+1 \\
& =\sum_{i=0}^{\infty}\left|\alpha_{1} q_{1}^{i}+\alpha_{2} q_{2}^{i}\right|+1,
\end{aligned}
$$

with coefficients $\alpha_{1}$ and $\alpha_{2}$ obtained directly from the matrices $K M$ and $M^{-1} B$. Reorganize each of these terms:

$$
\alpha_{1} q_{1}^{i}+\alpha_{2} q_{2}^{i}=\left(\alpha_{1}+\alpha_{2}\right) q_{1}^{i}+\alpha_{2}\left(q_{2}^{i}-q_{1}^{i}\right),
$$

considering that the two poles are positive real and ordered as follows:

$$
0 \leq q_{1} \leq q_{2}<1 .
$$

The first element is negative since we can observe from (38) and (59), and then from (16) that

$$
\begin{aligned}
\alpha_{1}+\alpha_{2} & =K B \\
& =k-k \cosh (\omega \tau)-k \lambda \omega \sinh (\omega \tau) \\
& =q_{1}+q_{2}-2 \cosh (\omega \tau)<0 .
\end{aligned}
$$

With the help of a computer algebra system, we can actually obtain that

$$
\begin{aligned}
& \alpha_{1}=\frac{1-q_{1}}{(k-1)\left(q_{1}-q_{2}\right)}\left(q_{1} q_{2}-1+k\left(1-q_{1}\right)\right), \\
& \alpha_{2}=\frac{1-q_{2}}{(k-1)\left(q_{2}-q_{1}\right)}\left(q_{1} q_{2}-1+k\left(1-q_{2}\right)\right) .
\end{aligned}
$$

Having $\alpha_{2}$ also negative would complete the proof. The fraction on the left is positive, so $\alpha_{2}$ has the same sign as the term on the right. When $\lambda \geq \omega^{-1}$, this term can be reformulated, using (15), as

$$
k\left(\cosh (\omega \tau)-\lambda \omega \sinh (\omega \tau)-q_{2}\right) \leq k\left(\mathrm{e}^{-\omega \tau}-q_{2}\right) .
$$

When $\lambda \leq \omega^{-1}$, the gray area satisfies $k \leq 1+\mathrm{e}^{-\omega \tau}$, so

$$
\begin{aligned}
q_{1} q_{2}-1+k\left(1-q_{2}\right) & \leq q_{2}^{2}-1+k\left(1-q_{2}\right) \\
& \leq\left(1-q_{2}\right)\left(k-1-q_{2}\right) \\
& \leq\left(1-q_{2}\right)\left(\mathrm{e}^{-\omega \tau}-q_{2}\right) .
\end{aligned}
$$

In both cases, this term is negative since at least one pole is greater or equal to $\mathrm{e}^{-\omega \tau}$ in the gray area, so $q_{2} \geq \mathrm{e}^{-\omega \tau}$.

\section{REFERENCES}

[1] C. Brasseur, A. Sherikov, C. Collette, D. Dimitrov, and P.-B. Wieber. A robust linear MPC approach to online generation of 3D biped walking motion. In IEEE-RAS International Conference on Humanoid Robots, pages 595-601, 2015.

[2] S. Caron, A. Kheddar, and O. Tempier. Stair climbing stabilization of the HRP-4 humanoid robot using whole-body admittance control. In IEEE International Conference on Robotics and Automation, 2019.

[3] J. Englsberger, G. Mesesan, A. Werner, and C. Ott. Torque-based dynamic walking - A long way from simulation to experiment. In IEEE International Conference on Robotics and Automation, pages 440-447, 2018.

[4] S. Feng, E. Whitman, X. Xinjilefu, and C. G. Atkeson. Optimizationbased full body control for the DARPA robotics challenge. Journal of Field Robotics, 32(2):293-312, 2015.

[5] T. Flayols, A. Del Prete, P. Wensing, A. Mifsud, M. Benallegue, and O. Stasse. Experimental evaluation of simple estimators for humanoid robots. In IEEE-RAS International Conference on Humanoid Robots, pages 889-895, 2017.

[6] R. Grandia, F. Farshidian, R. Ranftl, and M. Hutter. Feedback MPC for torque-controlled legged robots. In IEEE/RSJ International Conference on Intelligent Robots and Systems, 2019.

[7] B. Henze, M. A. Roa, A. Werner, A. Dietrich, C. Ott, and A. AlbuSchäffer. Experimental analysis of human-like behaviors in a humanoid robot: Quasi-static balancing using toe-off motion and stretched knees. In IEEE International Conference on Robotics and Automation, 2019.

[8] E. Jury. A simplified stability criterion for linear discrete systems. Proceedings of the IRE, 50(6):1493-1500, 1962.

[9] C. Mastalli, M. Focchi, I. Havoutis, A. Radulescu, S. Calinon, J. Buchli, D. G. Caldwell, and C. Semini. Trajectory and foothold optimization using low-dimensional models for rough terrain locomotion. In IEEE International Conference on Robotics and Automation, pages 10961103, 2017.

[10] D. Q. Mayne, M. M. Seron, and S. Raković. Robust model predictive control of constrained linear systems with bounded disturbances. Automatica, 41(2):219-224, 2005.

[11] K. Ogata. Discrete-time control systems, volume 8. Prentice-Hall Englewood Cliffs, NJ, 1995.

[12] D. Serra, C. Brasseur, A. Sherikov, D. Dimitrov, and P.-B. Wieber. A Newton method with always feasible iterates for nonlinear model predictive control of walking in a multi-contact situation. In IEEE-RAS International Conference on Humanoid Robots, pages 932-937, 2016.

[13] T. Sugihara. Standing stabilizability and stepping maneuver in planar bipedalism based on the best COM-ZMP regulator. In IEEE International Conference on Robotics and Automation, pages 1966-1971, 2009.

[14] N. A. Villa and P.-B. Wieber. Model predictive control of biped walking with bounded uncertainties. In IEEE-RAS International Conference on Humanoid Robots, pages 836-841, 2017.

[15] P.-B. Wieber, R. Tedrake, and S. Kuindersma. Modeling and control of legged robots. In Springer Handbook of Robotics, pages 1203-1234. Springer, 2016. 